\title{
Sample preparation for aberration-corrected microscopy of high-quality TEM specimens of advanced integrated circuits
}

\author{
C.S. Bonifacio ${ }^{1}$, M. Campin ${ }^{1}$, K. McIlwrath ${ }^{2}$, M. Ray ${ }^{1}$, and P.E. Fischione ${ }^{1}$ \\ ${ }^{1}$ E.A. Fischione Instruments, Inc., Export, PA, USA \\ 2 JEOL USA, Peabody, MA, USA
}

Advanced integrated circuits (ICs) are complex due to the fin field effect transistors (FinFETs), which comprise multigate transistors with the source/drain (S/D) channels (fins) surrounded by a threedimensional gate. State-of-the-art ICs are at the $10 \mathrm{~nm}$ and $7 \mathrm{~nm}$ nodes, with the later at the ramping stage of production[1]. At the $10 \mathrm{~nm}$ node, the S/D fins are $25 \%$ taller and $25 \%$ more closely spaced than $14 \mathrm{~nm}$ node technology [2]. Transmission electron microscopy (TEM) is a critical characterization tool for the semiconductor industry given the decreasing device size. $\mathrm{Ga}^{+}$focused ion beam (FIB) is frequently used for advanced IC TEM specimen preparation due to its rapid, site-specific sample preparation capabilities. However, FIB milling typically results in specimen artifacts, such as surface amorphization and $\mathrm{Ga}^{+}$implanted layers, both of which may limit analytical and high-resolution electron microscopy. Furthermore, $20 \mathrm{~nm}$ or less specimen thickness is required to characterize the 3D structures of the FinFET gate oxide in the TEM [3]. In this work, we present targeted, small spot $(<1 \mu \mathrm{m})$, low energy $\mathrm{Ar}^{+}$milling for reproducible specimen preparation of advanced ICs with specimen thicknesses of less than $20 \mathrm{~nm}$ that removes FIB-induced artifacts.

An Intel Broadwell $\mathrm{M}$ core processor was deprocessed and from it a cross-section specimen was created using the inverted method in a dual-beam FIB [Thermo Fisher]. Following $5 \mathrm{kV}$ milling in the FIB, specimen thicknesses that ranged from $80 \mathrm{~nm}$ to $200 \mathrm{~nm}$ were obtained. An ion milling system [Fischione Instruments] was used to iteratively mill the specimens at decreasing energies (from 900 to $500 \mathrm{eV}$ ). TEM and scanning TEM (STEM) characterization were performed before and after each ion milling step (the specimen was mounted on a TEM specimen holder that is compatible with both the $\mathrm{Ar}^{+}$ion mill and the TEM goniometer). Specimen thickness was determined by electron energy loss spectroscopy (EELS). The specimens were further imaged in a probe-corrected ARM200F [JEOL] after ion milling.

Preliminary results after ion milling resulted in electron-transparent specimens, as shown by the aberration-corrected dark field (DF) and bright field (BF) STEM images in Figures 1a-b, respectively. In Figures 1a-b, the atomic columns of $\mathrm{Si}$ in the fin and amorphous high-k (dark layer in DF-STEM; bright layer in BF-STEM) and work function setting material (bright layer in DF-STEM; dark layer in BFSTEM) above the fin were easily identified. EELS energy-filtered TEM (EFTEM) results (Figures 1c-d) indicated $t / \lambda$ ranging from 0.22 to 0.11 , which are equivalent to specimen thicknesses of 32.6 to $16.3 \mathrm{~nm}$. To confirm reproducibility, another set of specimens was iteratively milled by the $\mathrm{Ar}^{+} \mathrm{milling}$ system using the same parameters. The removal of the layers across the fin structure was determined by tilting the specimen in the TEM and acquiring DF-STEM images in between milling steps. Figure 2 shows the layer-by-layer removal across the fin structure - namely the gate oxide, spacer, and metal interconnects. The fin structure of the FinFET was targeted until the gate-oxide layers on both sides of the specimen were revealed (Figure 2c). Aberration-corrected microscopy and EELS thickness analysis are underway. 


\section{References:}

[1] M Lapedus, Semiconductor Engineering (2018), https://semiengineering.com/nodes-vs-node-lets/

[2] K. Mistry, Intel Corporation Technology and Manufacturing Day (2017).

[3] H. Feng et al., Proceedings of the $40^{\text {th }}$ International Symposium for Testing and Failure Analysis, (2014), p. 478.
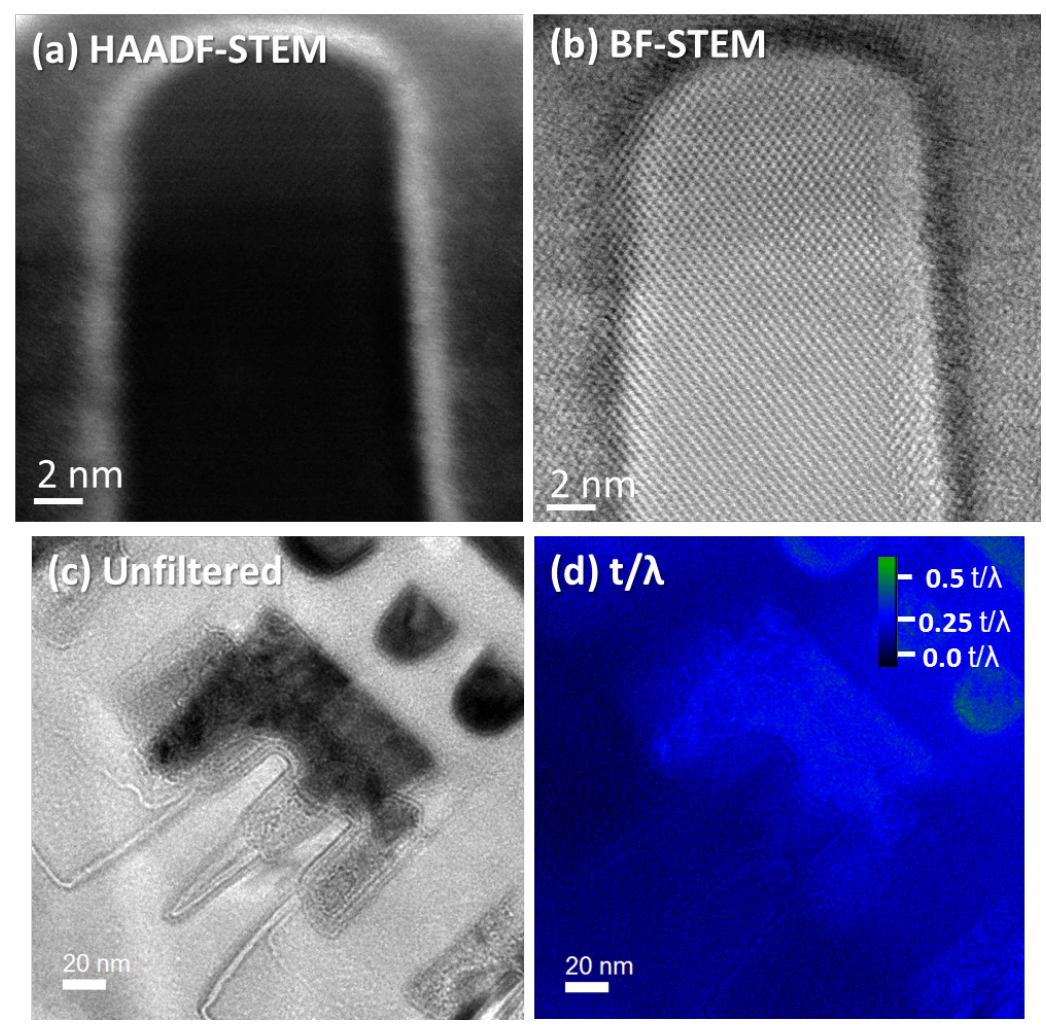

Figure 1. Aberration-corrected, high-angle, annular dark field (a) and bright field (b) STEM images of the FinFET structure after ion milling. Specimen thickness was calculated to be $25.2 \mathrm{~nm}$ based on a $\mathrm{MFP}=148.121 \mathrm{~nm}$ and $\mathrm{t} / \lambda=0.17$ derived from the unfiltered image (c) and EFTEM thickness map (d). The color is based on the $\mathrm{t} / \lambda$ scale.

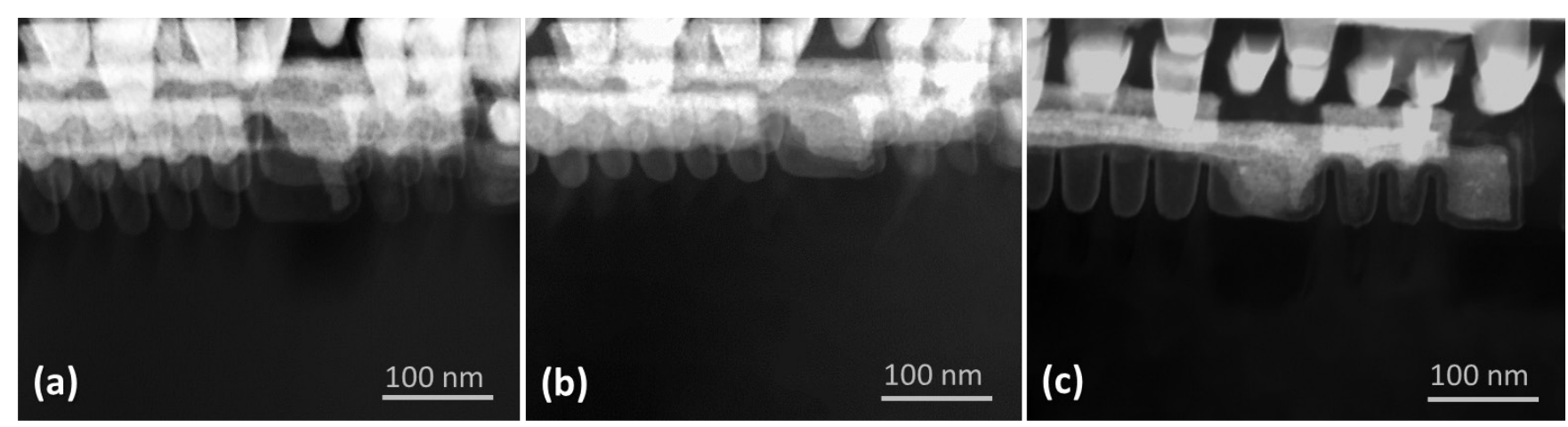

Figure 2. HAADF-STEM images of the specimen tilted at $\pm 27^{\circ}$ before $\mathrm{Ar}^{+}$milling (a) and following $700 \mathrm{eV}$ (b) and $500 \mathrm{eV}$ (c) $\mathrm{Ar}^{+}$milling. The gate oxide, spacer, and metal interconnect layers across the fin structure were removed layer-by-layer. 